\title{
(a) OPEN ACCESS \\ Effect of breathing on venous return during delayed cord clamping: an observational study
}

\author{
Emma Brouwer (1) , ${ }^{1}$ Ronny Knol, ${ }^{1,2}$ Annie Kroushev, ${ }^{3}$ Thomas Van Den Akker, ${ }^{4}$ \\ Stuart B Hooper, ${ }^{5,6}$ Arno A Roest, ${ }^{7}$ Arjan B te Pas ${ }^{1}$
}

${ }^{1}$ Neonatology, Leiden University Medical Center Willem Alexander Children's Hospital, Leiden, The Netherlands ${ }^{2}$ Neonatology, Erasmus MC, Rotterdam, The Netherlands ${ }^{3}$ Monash Women's, Monash Health, Clayton, Victoria,

Australia

${ }^{4}$ Obstetrics, Leids Universitair Medisch Centrum, Leiden, The Netherlands

${ }^{5}$ The Ritchie Centre, Hudson Institute of Medical Research, Clayton, Victoria, Australia ${ }^{6}$ Obstetrics and Gynaecology, Monash University, Clayton, Victoria, Australia

Pediatric Cardiology, Leiden University Medical Center Willem Alexander Children's Hospital, Leiden, The Netherlands

\section{Correspondence to} Emma Brouwer, Neonatology, Leiden University Medical Center Willem Alexander Children's Hospital, Leiden, ZA 2333, The Netherlands; e.brouwer@lumc.nl

Received 15 December 2020 Accepted 6 May 2021

\section{ABSTRACT}

Objective To investigate the effect of spontaneous breathing on venous return in term infants during delayed cord clamping at birth.

Methods Echocardiographic ultrasound recordings were obtained directly after birth in healthy term-born infants. A subcostal view was used to obtain an optimal view of the inferior vena cava (IVC) entering the right atrium, including both the ductus venosus (DV) and the hepatic vein (HV). Colour Doppler was used to assess flow direction and flow velocity. Recordings continued until the umbilical cord was clamped and were stored in digital format for offline analyses.

Results Ultrasound recordings were obtained in 15 infants, with a median (IQR) gestational age of 39.6 (39.0-40.9) weeks and a birth weight of 3560 (31954205) g. Flow was observed to be antegrade in the DV and HV in $98 \%$ and $82 \%$ of inspirations, respectively, with flow velocity increasing in $74 \%$ of inspirations. Retrograde flow in the DV was observed sporadically and only occurred during expiration. Collapse of the IVC occurred during $58 \%$ of inspirations and all occurred caudal to the DV inlet (100\%).

Conclusion Spontaneous breathing was associated with collapse of the IVC and increased antegrade DV and HV flow velocity during inspiration. Therefore, inspiration appears to preferentially direct blood flow from the DV into the right atrium. This indicates that inspiration could be a factor driving placental transfusion in infants.

\section{INTRODUCTION}

It is well established that delayed cord clamping (DCC) is beneficial to preterm and term infants ${ }^{1-3}$ and is therefore currently recommended for all infants not needing immediate resuscitation. ${ }^{4}$ However, the underlying mechanisms behind these benefits are unclear. Studies have demonstrated that delaying cord clamping until after breathing onset maintains cardiac output throughout the fetal to neonatal transition by ensuring continued umbilical venous return to the heart. Similarly, DCC enables the net movement of blood volume from the placenta to the neonate (placental transfusion), which is also dependent on sustaining umbilical venous return into the infant. ${ }^{1-3}$ However, despite placental transfusion being verified in several clinical studies, the underlying physiological mechanisms remain elusive. ${ }^{5-7}$ Possible mechanisms for placental transfusion include the effects of gravity and uterine contractions. ${ }^{89}$ However, the effect of gravity has recently been refuted in experimental and human studies, ${ }^{10} 11$ and experimental studies

\section{What is already known on this topic?}

- Delayed cord clamping is beneficial for both term-born and preterm-born infants.

- Delaying cord clamping until after breathing has commenced maintains cardiac output during neonatal transition by ensuring umbilical venous return.

\section{What this study adds?}

Blood flow in the ductus venosus and hepatic vein is predominantly antegrade and increases during inspiration directly after birth.

- Inspiration during delayed cord clamping potentially generates a preferential ductus venosus blood flow by obstructing the inferior vena cava.

have shown that uterine contractions markedly reduce (rather than increase) umbilical venous blood flow (UVBF). ${ }^{12} 13$

It has also been suggested that spontaneous breathing during DCC enhances umbilical venous return by generating subatmospheric intrathoracic pressures that increase umbilical venous inflow and reduce umbilical arterial outflow from the infant. This could explain the maintenance of cardiac output and net increase in neonatal blood volume when DCC occurs after breathing onset. ${ }^{13}{ }^{14}$ Indeed, studies using residual placental blood volumes as a measure for placental transfusion have shown that the onset of respiration prior to cord clamping reduces residual placental blood volume. ${ }^{1516}$ Ultrasound studies of umbilical blood flow in human fetuses have also shown that fetal breathing movements (FBM) are associated with distention of the umbilical vein and an increase in UVBF. In contrast, the inspiratory phase was associated with a concentric reduction in the cross section of the inferior vena cava (IVC), resulting in significantly reduced flow. ${ }^{17} 18$ We previously used ultrasonography during DCC after birth to show that umbilical blood flow continues much longer than assumed prior to that study and that spontaneous breathing influences UVBF. However, the exact relationship between inspiration/expiration and umbilical blood flow could not be defined since inspiration and expiration were not specifically recorded. ${ }^{14}$ 


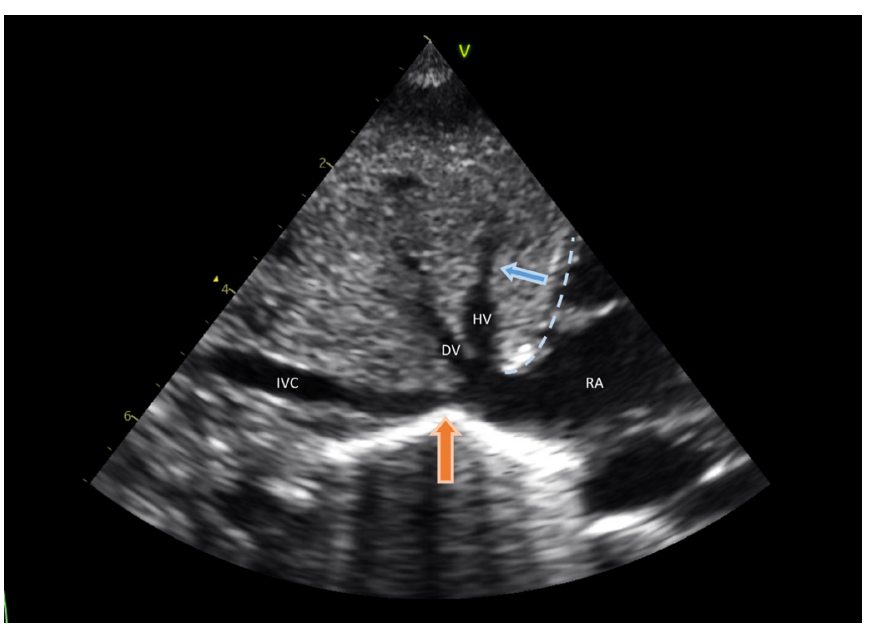

Figure 1 Subcostal view of the inferior vena cava (IVC) entering the right atrium (RA) with both the ductus venosus (DV) and the hepatic vein (HV) visible. This subcostal view represents the anatomical composition as was observed in all infants. Dashed line: location of the diaphragm. Blue arrow: direction of diaphragm movement with inspiration. Orange arrow: location of IVC collapse, directly caudal to the DV inlet.

We recently examined the effect of spontaneous breathing on UVBF in newborn lambs during DCC and surprisingly found that inspiration causes UVBF to decrease or cease. ${ }^{19}$ This was attributed to constriction of the IVC at the level of the diaphragm during inspiration, as previously observed in human fetuses with antenatal ultrasound. ${ }^{18}$ In fetal sheep, the umbilical venous blood traverses the ductus venosus (DV) and passes into the IVC before entering the thorax. It then passes along a $3-4 \mathrm{~cm}$ segment of the intrathoracic IVC before entering the right atrium (RA) or foramen ovale. As such, constriction of the IVC at the diaphragm was thought to also reduce UVBF through the DV. ${ }^{20}{ }^{21}$ However, in humans the abdominal IVC ends in a funnel-like venous structure, which also contains the orifices of the hepatic vein (HV) and the DV. This subdiaphragmatic venous vestibulum is located directly below and at the level of the diaphragm, before entering the RA (figure 1). ${ }^{22}$ It is unclear whether inspiration interferes with UVBF in humans as it does in sheep based on this anatomical difference. We hypothesise that in humans, inspiration causes an increase in UVBF, as occurs antenatally, at the expense of flow through the IVC, thereby prioritising umbilical venous return to the heart directly after birth. Our aim was to investigate the effect of spontaneous breathing on umbilical venous return by measuring both blood flow in the DV, as a measure of UVBF, and the diameter of the IVC.

\section{METHODS}

A prospective observational study was performed from February 2018 until September 2019 at Leiden University Medical Center.

\section{Study population}

Infants born vaginally between 37 and 42 weeks of gestational age were eligible if they were born after an uncomplicated and low-risk pregnancy to a multiparous woman. Infants were excluded if they were known or suspected to have congenital heart or pulmonary conditions, if the infant needed respiratory support or if additional oxygen during transition was required. Written parental consent was obtained prior to birth.

\section{Equipment and procedures}

Directly after birth, infants were dried and placed on the woman's chest with an intact umbilical cord, as per standard of care. Infants were placed in a supine position before ECG leads were attached and ultrasound measurements commenced. If placement of the infant in a supine position was determined to be undesirable by the midwife for any reason, if the umbilical cord was too short, or if one of the parents felt uncomfortable with repositioning the baby, the study procedure was discontinued and the infant excluded from the study.

Recordings were performed using a Vivid S6 or Vivid S60 (GE Healthcare) echocardiographic ultrasound machine with a neonatal/paediatric $6 \mathrm{~S}$ probe. The probe was placed gently over the subcostal region and slowly rotated and tilted until an optimal view of the IVC entering the RA was obtained, which also included both the DV and the HV (figure 1). Recordings continued until the umbilical cord was clamped, at the discretion of the midwife, and recordings were stored in digital format for offline analyses. In the event that parents withdrew consent, the woman or the neonate needed medical support, or for any other reason the umbilical cord needed to be clamped prior to finishing the ultrasound examination, measurements were discontinued immediately.

\section{Measurements}

\section{Respiration}

Spontaneous breathing was observed by diaphragm movements visible in all recordings. Caudal or caudal-anterior movement of the diaphragm was defined as inspiration, whereas cranial or cranial-posterior movement was defined as expiration (figure 1). Both inspiration and expiration were measured separately and as part of a complete respiration cycle including inspiration and expiration within one recording. If no diaphragm movement was visible, this was considered a 'respiratory pause', which may occur after both inspiration (expiratory hold; helps maintain functional residual capacity and clear lung liquid) and expiration (postexpiratory hold). ${ }^{23}$

\section{Ductus venosus}

A subcostal view was used to visualise both the DV and the HV. Colour Doppler was then used to visualise flow direction in both vessels during inspiration, expiration and respiratory pauses. Flow velocity in the DV and HV was assessed with colour Doppler using M-mode. Increase of flow was evaluated as a visual increase of colour in the respective vessel (figure 2).

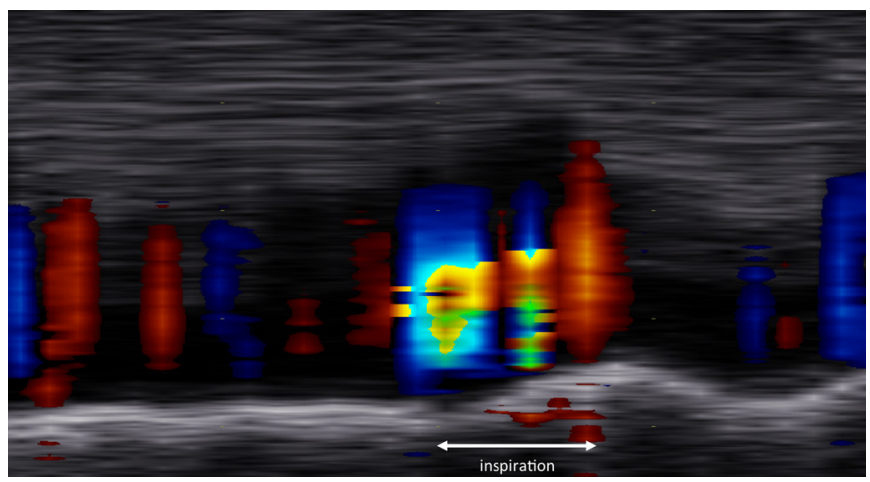

Figure 2 Example of a flow pattern in the hepatic vein during inspiration. There is antegrade flow (blue, direction towards the infant) with an increasing velocity during inspiration. The arrow indicates inspiration by diaphragm movement. 
Table 1 Baseline characteristics $(\mathrm{N}=15)$

\begin{tabular}{lc}
\hline Maternal characteristics & \\
Maternal age & $33(30-35)$ \\
\hline Gravidity & $3(2-4)$ \\
\hline Parity & $3(2-3)$ \\
\hline Neonatal characteristics & \\
Gestational age, weeks & $39.6(39.0-40.9)$ \\
\hline Female & $7(46.7)$ \\
\hline Birth weight, g & $3560(3195-4205)$ \\
\hline Apgar 1 min & $9(9-9)$ \\
\hline Apgar 5 min & $10(10-10)$ \\
\hline Apgar 10 min & $10(10-10)$ \\
\hline
\end{tabular}

Data presented as median (IQR) or $\mathrm{n}(\%)$.

Flow direction was defined as antegrade (towards the infant) if the majority of flow during a respiratory phase was antegrade, which was assessed with colour Doppler using M-mode. Flow was defined as retrograde if the majority of flow during a respiratory phase was retrograde (away from the infant).

\section{Inferior vena cava}

Colour Doppler flow measurements in the IVC were unavailable due to the $90^{\circ}$ angle of the subcostal view. IVC diameter (IVCDia) was measured using standard two-dimensional grey scale images of the subcostal view. Change in IVC-Dia was measured for every recorded inspiration movement, when the IVC was visible over the entire length. If a change in IVC-Dia was present during inspiration, the location of this diameter change was noted as either cranial or caudal to the DV inlet into the subdiaphragmatic venous vestibulum or at the DV.

\section{Statistical analysis}

Data are presented as mean $( \pm S D)$, median $(\mathrm{IQR})$ or number (\%), where appropriate. SPSS V.24.0 software for Windows was used for the database and statistics.

\section{RESULTS}

A total of 17 infants were included in the study, although ultrasound measurements could not be performed in 2 infants due to complications at birth, that is, shoulder dystocia and postpartum haemorrhage. Thus, ultrasound recordings were obtained in 15 infants, with a median (IQR) gestational age of 39.6 (39.0-40.9) weeks and a birth weight of 3560 (3195-4205) g (table 1). Measurements were obtained over a median time of 06:35 (05:12-07:56) min.

Using direct assessment of diaphragm movement, we were able to observe 129 inspirations and 118 expirations, of which 104 respiratory cycles were complete and thereby included both inspiration and expiration, with or without a respiratory pause, within one recording. The median recorded respiratory cycles per infant were 5 (3-11). However, due to the short duration of the recordings, it was not possible to distinguish between respiratory pauses that occurred after inspiration or after expiration. Therefore, all observations of 'respiratory pauses' were combined.

\section{Flow in DV and HV}

DV and HV flow was observed in all infants. Antegrade flow in the DV was observed in 56 of $57(98 \%)$ inspirations, 27 of 46 (59\%) expirations and 33 of 41 (81\%) respiratory pauses.

\begin{tabular}{lccc} 
Table 2 & Flow direction in the DV and HV during respiration \\
\hline & $\begin{array}{l}\text { Antegrade } \\
\text { flow }\end{array}$ & $\begin{array}{l}\text { Retrograde } \\
\text { flow }\end{array}$ & $\begin{array}{l}\text { High-frequency } \\
\text { bidirectional flow }\end{array}$ \\
\hline DV & & & \\
Inspiration $(n=57)$ & 98 & 0 & 2 \\
Expiration $(n=46)$ & 59 & 4 & 37 \\
Respiratory pause $(n=41)$ & 81 & 0 & 19 \\
HV & & & \\
Inspiration $(n=61)$ & 82 & 0 & 18 \\
Expiration $(n=72)$ & 25 & 13 & 62 \\
Respiratory pause $(n=52)$ & 17 & 0 & 83
\end{tabular}

Data presented as \%.

DV, ductus venosus; HV, hepatic vein.

Retrograde flow occurred sporadically in the DV and was only observed in 2 of 46 (4\%) expirations (table 2).

Antegrade flow in the HV was observed in 50 of 61 (82\%) inspirations, whereas in 45 of $72(62 \%)$ expirations and in 43 of $52(83 \%)$ respiratory pauses an alternating bidirectional (antegrade/retrograde) flow pattern occurred at a high frequency, which was approximately twice the heart rate (figure 3 ). Retrograde flow in the $\mathrm{HV}$ also occurred sporadically and was only observed in 9 of $72(13 \%)$ expirations.

Evaluation of flow increase in either the DV, the HV or in both vessels shows that antegrade flow velocity increased during 56 of $76(74 \%)$ inspirations and during 12 of 50 (24\%) respiratory pauses. Increases in retrograde flow velocity occurred in $9 \%(7$ of 79) of expirations.

\section{Diameter of IVC}

IVC-Dia could be reliably measured in all infants. The IVC collapsed in 66 of 101 inspirations and respiratory pauses, but in only 7 of $67(10 \%)$ expirations (table 3). This collapse always (72 of $72,100 \%)$ occurred caudal to the DV inlet into the subdiaphragmatic venous vestibulum. Of these constrictions, 64 of $72(89 \%)$ occurred directly below the DV inlet (figure 1), with a median change in diameter of $2.6(1.9-3.5) \mathrm{mm}$ (from $3.6(2.7-4.5) \mathrm{mm}$ to $0.0(0.0-2.1) \mathrm{mm})$, whereas in $11 \%$ the IVC constriction occurred further upstream. On these occasions, the

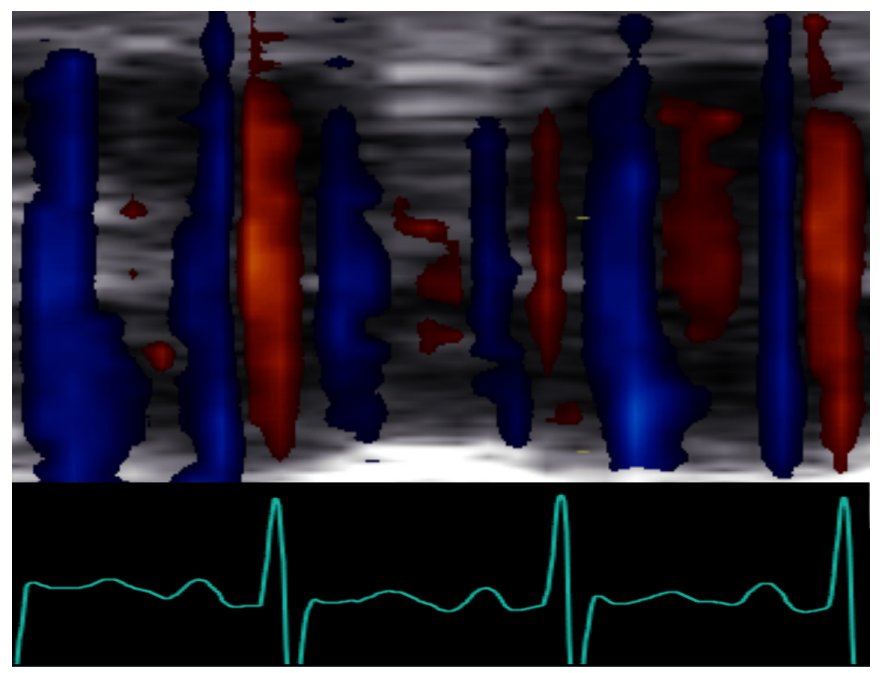

Figure 3 Cross section of the hepatic vein over time using colour Doppler M-mode. Blood flow direction alternates between antegrade (blue) and retrograde (red) with a high frequency and corresponding ECG signal. 


\begin{tabular}{llll}
\hline Table 3 & Collapse of the inferior vena cava during respiration \\
\hline & $\begin{array}{l}\text { Inspiration } \\
(\mathbf{n}=69)\end{array}$ & $\begin{array}{l}\text { Expiration } \\
(\mathbf{n}=67)\end{array}$ & $\begin{array}{l}\text { Respiratory pause } \\
(\mathbf{n}=32)\end{array}$ \\
\hline Collapse & $40(58)$ & $7(10)$ & $26(81)$ \\
Complete & $23(58)$ & $5(71)$ & $5(19)$ \\
Incomplete & $17(42)$ & $2(29)$ & $21(81)$ \\
No collapse & $29(42)$ & $60(90)$ & $6(19)$ \\
\hline
\end{tabular}

Data are presented as $\mathrm{n}(\%)$.

collapse occurred $10(9.3-10.2) \mathrm{mm}$ caudal to the DV inlet into the vestibulum, with a median change in diameter of 2.2 (2.02.8 ) $\mathrm{mm}$ (from 4.1 (3.4-5.3) $\mathrm{mm}$ to $2.1(1.6-2.5) \mathrm{mm}$ ).

\section{DISCUSSION}

We used ultrasound to assess the effect of breathing on DV and HV blood flow in human infants at birth prior to umbilical cord clamping. We observed that breathing was associated with an increase in antegrade blood flow in both the DV and the HV during inspiration. While blood flow continued in antegrade direction in the DV during both expiration and respiratory pauses, blood flow in the HV became bidirectional and alternated at high frequency (approximately twice the heart rate). Furthermore, inspiration was associated with collapse of the IVC, resulting in an obstructed IVC flow as has previously been described in human fetuses. ${ }^{18}$ These observations indicate that inspiration facilitates and prioritises umbilical venous return from the placenta into the heart during DCC. Thus, breathing could contribute to placental transfusion during DCC, and as such the presence or absence of breathing may be a complicating factor in previous placental transfusion studies. ${ }^{24}$

Our observations of the effect of inspiration on DV and HV in term infants are in line with previous antenatal ultrasound studies of breathing movements in humans. Kiserud et $a l^{25}$ demonstrated a 10 -fold increase in pressure gradient between the DV and IVC during inspiration. Nyberg et $a l^{17}$ showed that umbilical blood flow increased by $42 \%$ during inspiration, when measured at the placental end of the umbilical cord. Our current findings confirm those of a previous study in which we observed increases and decreases in umbilical blood flow that appeared to be related to breathing. ${ }^{14}$ However, when we examined the underlying physiology using a spontaneously breathing lamb model, we were surprised to find the opposite effect, whereby UVBF decreased during inspiration. ${ }^{19}$ In contrast to humans, lambs have a lengthy $(3-4 \mathrm{~cm})$ intrathoracic segment of the IVC and so it is possible that this difference in anatomy is responsible for the opposite effects on UVBF during breathing. That is, as the DV joins the IVC before it enters the chest in fetal sheep, the effect of diaphragmatic contraction on IVC blood flow also reduces DV flow. If this is correct, it is likely that the highly significant benefits of physiological-based cord clamping (lung aeration and breathing onset before cord clamping) that have been demonstrated in sheep are even greater in humans.

Presumably, a complete collapse of the IVC directly below the DV inlet during inspiration causes a cessation of IVC flow. When combined with an increase in antegrade flow in both the DV and the HV, this would result in preferential blood flow streaming from the DV into the RA. This could explain the increase in neonatal blood volume during DCC in spontaneously breathing infants. It would also explain why infants who breathe prior to cord clamping have less residual placental blood volume, that is, more placental transfusion. ${ }^{16}$
The IVC was observed to collapse caudal to the DV inlet during inspiration, which is a similar location to the IVC collapse observed previously during FBM, which was associated with a concentric reduction in IVC-Dia. It is possible, however, that our results represent an underestimation of IVC collapse. Namely, we excluded recordings in which the IVC was not visible over the entire length to correct for the potential influence of partial volume effect/movement of the IVC out of the imaging plane and to ensure an optimal and accurate assessment of the IVC collapse. However, excluding these recordings could have influenced our results and led to an underestimation of the true relationship between IVC collapse and inspiration.

Interestingly, we found a high-frequency bidirectional flow pattern in the $\mathrm{HV}$ that is unlikely to be respiratory in origin as it was most commonly observed during respiratory pauses or expiration. However, as the measured frequency was approximately twice the heart rate, we speculate that it is due to atrial pressure changes during the cardiac cycle resulting in retrograde pressure waves within the HV. This is evidenced by aligning the individual components of the ECG to the observed flow patterns in the $\mathrm{HV}$ (figure 3). Atrial pressures increase twice per cardiac cycle, once during atrial contraction and again at maximum atrial filling immediately before the atrioventricular valves open. ${ }^{26}$ Indeed, retrograde flow in the $\mathrm{HV}$ has been described during atrial contraction (a-wave), but also during maximal atrial filling (v-wave). ${ }^{27}$ It is possible that the immaturity (and therefore stiffness) of the preterm heart increases this effect, resulting in retrograde flow in the HV twice per cardiac cycle. As this high-frequency bidirectional flow pattern was most commonly seen during respiratory pause and expiration, it is likely that this cardiac effect on HV blood flow is diminished by the negative intrathoracic pressure created during inspiration.

Retrograde flow in either the DV or the HV was observed sporadically and only during expiration. While retrograde flow in the HV has been described during atrial contraction and atrial filling, ${ }^{26}{ }^{27}$ antenatal presence of retrograde DV flow is often an indication of placental insufficiency and cardiac compromise in infants with severe growth restriction. ${ }^{28}$ Unfortunately, antenatal DV Doppler measurements were unavailable in this study. However, as we only included healthy infants of low-risk pregnancies, it is highly unlikely that the retrograde flow observed in this study was an extension of antenatal flow patterns based on placental insufficiency and/or cardiac compromise.

Obtaining these measurements directly at birth has proven to be challenging and we were only able to collect data from a small group of healthy term infants. The infant was placed on the mother's chest and measurements may have been influenced by movement from the infant itself, or by repositioning or stimulation of the infant by the parents and caregivers, which limited the number of reliable measurements. Although a larger study would be needed to confirm our findings, the observations made in this small group of infants were quite consistent, making generalisation of our results plausible.

\section{CONCLUSION}

Blood flow in both the DV and the HV is predominantly antegrade and increases during inspiration in healthy term-born infants. Interestingly, collapse of the IVC during inspiration consistently occurred caudal to the DV inlet and therefore had minimal impact on umbilical venous return, unlike in sheep. Instead, breathing during DCC tends to preferentially direct umbilical blood flow through the DV and into the RA. As such spontaneous breathing may partially explain the net increase 
in neonatal blood volume by providing the driving force for placental transfusion.

Contributors EB, RK, AAR, SBH and ABtP wrote the ethics application. EB, $A A R$ and $A B t P$ participated in the study design and coordination, collected and analysed the data, and reviewed the literature. RK, AK, TVDA and SBH participated in the study design. EB wrote the first draft and submitted the article. All authors participated in reviewing the data and editing the manuscript. All authors have read and approved the final manuscript. AAR and ABtP share last authorship.

Funding $A B t P$ was a recipient of a Vidi grant, The Netherlands Organisation for Health Research and Development (ZonMw), part of the Innovational Research Incentives Scheme Veni-Vidi-Vici (NWO Vidi 2015/2016).

Competing interests None declared.

Patient consent for publication Not required.

Ethics approval This study was approved by the Leiden University Medical Center Institutional Review Board (IRB p17.139).

Provenance and peer review Not commissioned; externally peer reviewed.

Data availability statement Data are available upon reasonable request.

Open access This is an open access article distributed in accordance with the Creative Commons Attribution Non Commercial (CC BY-NC 4.0) license, which permits others to distribute, remix, adapt, build upon this work non-commercially, and license their derivative works on different terms, provided the original work is properly cited, appropriate credit is given, any changes made indicated, and the use is non-commercial. See: http://creativecommons.org/licenses/by-nc/4.0/.

ORCID iD

Emma Brouwer http://orcid.org/0000-0002-4022-1567

\section{REFERENCES}

1 McDonald SJ, Middleton P, Dowswell T, et al. Effect of timing of umbilical cord clamping of term infants on maternal and neonatal outcomes. Cochrane Database Syst Rev 2013;5:Cd004074.

2 Fogarty M, Osborn DA, Askie L, et al. Delayed vs early umbilical cord clamping for preterm infants: a systematic review and meta-analysis. Am J Obstet Gynecol 2018;218:1-18

3 Rabe H, Diaz-Rossello JL, Duley L, et al. Effect of timing of umbilical cord clamping and other strategies to influence placental transfusion at preterm birth on maternal and infant outcomes. Cochrane Database Syst Rev 2012;117:Cd003248.

4 Wyllie J, Bruinenberg J, Roehr CC, et al. European resuscitation Council guidelines for resuscitation 2015: section 7. resuscitation and support of transition of babies at birth. Resuscitation 2015;95:249-63.

5 Yao AC, Moinian M, Lind J. Distribution of blood between infant and placenta after birth. Lancet 1969;2:871-3.

6 Law GR, Cattle B, Farrar D, et al. Placental blood transfusion in newborn babies reaches a plateau after $140 \mathrm{~S}$ : further analysis of longitudinal survey of weight change. SAGE Open Med 2013;1:205031211350332.
7 Mercer JS, Erickson-Owens DA, Collins J, et al. Effects of delayed cord clamping on residual placental blood volume, hemoglobin and bilirubin levels in term infants: a randomized controlled trial. J Perinato/ 2017;37:260-4.

8 Yao AC, Lind J. Effect of gravity on placental transfusion. Lancet 1969:2:505-8.

9 Yao AC, Hirvensalo M, Lind J. Placental transfusion-rate and uterine contraction. Lancet 1968:1:380-3.

10 Hooper SB, Crossley KJ, Zahra VA, et al. Effect of body position and ventilation on umbilical artery and venous blood flows during delayed umbilical cord clamping in preterm lambs. Arch Dis Child Fetal Neonatal Ed 2017;102:F312-9.

11 Vain NE, Satragno DS, Gorenstein AN, et al. Effect of gravity on volume of placental transfusion: a multicentre, randomised, non-inferiority trial. Lancet 2014;384:235-40.

12 Westgate JA, Wibbens B, Bennet $L$, et al. The intrapartum deceleration in center stage: a physiologic approach to the interpretation of fetal heart rate changes in labor. Am J Obstet Gynecol 2007;197:236.e1-236.e11.

13 Hooper SB, Binder-Heschl C, Polglase GR, et al. The timing of umbilical cord clamping at birth: physiological considerations. Matern Health Neonatol Perinatol 2016;2:4.

14 Boere I, Roest AAW, Wallace E, et al. Umbilical blood flow patterns directly after birth before delayed cord clamping. Arch Dis Child Fetal Neonatal Ed 2015;100:F121-5.

15 Redmond A, Isana S, Ingall D. Relation of onset of respiration to placental transfusion. Lancet 1965:1:283-5.

16 Kjeldsen J, Pedersen J. Relation of residual placental blood-volume to onset of respiration and the respiratory-distress syndrome in infants of diabetic and nondiabetic mothers. Lancet 1967;1:180-4.

17 Nyberg MK, Johnsen SL, Rasmussen S, et al. Fetal breathing is associated with increased umbilical blood flow. Ultrasound Obstet Gynecol 2010;36:718-23.

18 Nyberg MK, Johnsen SL, Rasmussen S, et al. Hemodynamics of fetal breathing movements: the inferior vena cava. Ultrasound Obstet Gynecol 2011;38:658-64.

19 Brouwer E, Te Pas AB, Polglase GR, et al. Effect of spontaneous breathing on umbilical venous blood flow and placental transfusion during delayed cord clamping in preterm lambs. Arch Dis Child Fetal Neonatal Ed 2020;105:26-32.

20 Rudolph AM. Distribution and regulation of blood flow in the fetal and neonatal lamb Circ Res 1985;57:811-21.

21 Kiserud T. The ductus venosus in the human fetus: an ultrasonographic study of its functional anatomy, normal blood flow velocity and its changes during fetal disease. Acta Obstetrica et Gynecologica Scandinavica 1994:74:583-5.

22 Huisman TW, Gittenberger-de Groot AC, Wladimiroff JW. Recognition of a fetal subdiaphragmatic venous vestibulum essential for fetal venous Doppler assessment. Pediatr Res 1992;32:338-41

23 te Pas $A B$, Wong C, Kamlin COF, et al. Breathing patterns in preterm and term infants immediately after birth. Pediatr Res 2009;65:352-6.

24 Tarnow-Mordi W, Morris J, Kirby A, et al. Delayed versus immediate cord clamping in preterm infants. N Engl J Med 2017;377:2445-55.

25 Kiserud T, Hellevik LR, Eik-Nes SH, et al. Estimation of the pressure gradient across the fetal ductus venosus based on Doppler velocimetry. Ultrasound Med Biol $1994 ; 20: 225-32$

26 Tansey EA, Montgomery LEA, Quinn JG, et al. Understanding basic vein physiology and venous blood pressure through simple physical assessments. Adv Physiol Educ 2019:43:423-9.

27 Scheinfeld MH, Bilali A, Koenigsberg M. Understanding the spectral Doppler waveform of the hepatic veins in health and disease. Radiographics 2009:29:2081-98.

28 Baschat AA, Hecher K. Fetal growth restriction due to placental disease. Semin Perinatol 2004:28:67-80 